\title{
Nachruf auf Bernhard Blanke (6.3.1941-5.8.2014)
}

Am 5. August 2014 verstarb der langjährige Mitherausgeber des Leviathan nach einer kurzen und schweren Krankheit. In Zusammenarbeit mit seinen Mitherausgebern hat er das Profil der Zeitschrift stark geprägt. Von 1984 bis 1998 war er Herausgeber, danach bis zu seinem Tod im Beirat, und man kann ohne Übertreibung sagen, dass ihn die Tätigkeit beim Leviathan den größten Teil seines akademischen Lebens begleitet hat. Das Zeitschriftenmachen war ein Lebenselixier für ihn; seine Emeritierung verstand er nicht als »Ruhestand «, sondern er gründete eine neue interdisziplinäre Zeitschrift, der moderne staat, in der alle Aspekte der modernen Staatlichkeit diskutiert werden und die sich zum Zentrum der Staats- und Verwaltungsdiskussion in Deutschland entwickelt hat. Mit der Rückkehr zu Staatsfragen schließt sich in gewisser Weise ein Kreis, denn mit Fragen des Staates hat er seine wissenschaftliche Karriere begonnen.

Nach dem Studium der Rechts- und Politikwissenschaft in Tübingen, London und Berlin promovierte er an der Freien Universität Berlin im Jahr 1975. 1978 kam er als akademischer Rat an die Technische Universität Hannover, die heutige Leibniz Universität, erhielt dann einen Ruf an das dortige Institut für Politikwissenschaft, nachdem ein zunächst angestrengtes Berufsverbotsverfahren gegen ihn eingestellt worden war. In einer Anhörung vor einer Kommission des Niedersächsischen Ministeriums des Inneren 1975 vor seiner Verbeamtung gelang es ihm, Zweifel an seiner Verfassungstreue auszuräumen.

Sein Eintritt in die (Politik-)Wissenschaft war die mit Ulrich Jürgens und Hans Kastendiek zusammen verfasste, zweibändige Kritik der Politischen Wissenschaft. Analysen von Politik und Ökonomie in der bürgerlichen Gesellschaft (Frankfurt a. M. 1975). Hier war er für das wichtige Kapitel »Theorien zum Verhältnis von Staat und Gesellschaft. Zum Problem der Legitimation politischer Herrschaft in der bürgerlichen Gesellschaft « verantwortlich. Diese Kritik der politischen Wissenschaft war eine Replik auf den 1972 erschienenen Band Politikwissenschaft. Eine Einführung in ihre Probleme, der von Gisela Kress und Dieter Senghaas herausgegeben worden war und ebenfalls eine linke Kritik der bürgerlichen Politikwissenschaften anstrebte. In dieser Kritik publizierten neben den Herausgebern Autoren wie Claus Offe, Wolf-Dieter Narr, Walter Euchner, Joachim Hirsch und andere.

Blanke hatte bereits in seiner Diplomarbeit von 1971 seinen Gegenstand gefunden, in der er über die "Parlamentsreform in der Bundesrepublik " schrieb: die Staatstheorie, die er dann systematisch ausbaute. In der Kritik der Politischen Wissenschaft hatte er alle wichtigen »bürgerlichen « Theorien über das Verhältnis von Staat und Gesellschaft aufgearbeitet beziehungsweise kritisiert und insbesondere die Weimarer Staatsrechtslehre rekonstruiert und auf das Problem des Klassenstaates 
fokussiert. Später folgten dann Arbeiten zur marxistischen Staatsableitung, in denen er sich vor allem mit ähnlich gelagerten Versuchen von Claus Offe auseinandersetzte. Auch wichtige Arbeiten zum nationalsozialistischen Staat beziehungsweise zu Herrschaftsmechanismen des NS-Regimes schlossen sich an.

Nach seinem Ruf an die Universität Hannover folgten Arbeiten zum Wohlfahrtsstaat, insbesondere zur Regulierung des Arbeitsmarktes, zur Armutspolitik und später auch zur Gesundheitspolitik. Diese Forschungen fanden in dem von ihm 1991 gegründeten gemeinnützigen Institut für Sozialpolitik und Stadtforschung statt, das später in Institut für Sozialpolitik und Policy Studies umbenannt wurde. Neben drittmittelfinanzierten Forschungsprojekten wurde hier auch angewandte Politikforschung betrieben, die auf einen Brückenschlag zur praktischen Politik in Form von Politikberatung abzielte. Zu den wichtigsten Projekten gehörten unter anderem die "Sozialbilanz Niedersachsen ", die für die Staatskanzlei erstellt wurde, und der »Dialog Soziales Niedersachsen ", womit die Verbesserung vor allem der kommunalen Sozialpolitik angestrebt wurde. Das Institut ermöglichte vielen Politik- und SozialwissenschaftlerInnen eine Beschäftigung jenseits der Universität, aber gleichzeitig in enger Verbindung mit ihr. Auch konnten so zeitliche Restriktionen der universitären Beschäftigungsregeln umgangen werden.

Blanke war auch dem Fach und seiner Berufsvereinigung eng verbunden. Er war von 1975 bis 1977 und von 1983 bis 1988 als Mitglied im Beirat und von 1988 bis 1991 im Vorstand der Deutschen Vereinigung für Politische Wissenschaft (DVPW). 1991 organisierte er den DVPW-Kongress in Hannover, und etliche Sektions- und Arbeitsgruppentagungen der DVPW fanden auf sein Betreiben in Hannover statt.

Auch wenn er nie eine »Schule « gegründet beziehungsweise betrieben hat, sind viele seiner Mitarbeiter erfolgreiche Politikwissenschaftler geworden. Die Keimzelle hierfür war sicherlich das Institut und später die Abteilung »Sozialpolitik und Public Policy « des Instituts für Politikwissenschaft. Statt Schulenbildung hat er seinen MitarbeiterInnen Räume für die Forschung zur Verfügung gestellt und Platz für die Entwicklung eigener Ideen gelassen. Ein weiteres Ergebnis dieser innovativen Atmosphäre war seine Idee des »Aktivierenden Staates «, die in der ersten Legislaturperiode der rot-grünen Koalitionsregierung im Bund einen gewissen Einfluss auf die Regierungspolitik hatte. Diese Idee war der Ausfluss seines immerwährenden Nachdenkens über Reformspielräume staatlicher Institutionen, die Spannbreite der Modernisierung der Staatsverwaltung und auch neuer Formen der Verantwortungsteilung zwischen Staat und Gesellschaft. Die zu seiner Verabschiedung von der Universität im Jahr 2008 zu seinen Ehren durchgeführte Festveranstaltung beschäftigte sich folgerichtig mit »Academics, Think Tanks und Consultants: Zum Verhältnis von Wissenschaft, Politik und Politikberatung im Feld der Staatsmodernisierung «. Hier standen vor allem die Möglichkeiten der Politikwissenschaft als praktische und beratende Wissenschaft zur Debatte, und reflektiert wurden die Möglichkeiten, aber auch Grenzen der wissenschaftlichen Politikberatung.

In den letzten Jahren hat ihn dann seine politische Vergangenheit überraschend eingeholt. Der Schriftsteller Peter Schneider hatte in einem Interview in der Frankfurter Allgemeinen Zeitung vom 3. Juni 2009 erwähnt, dass die Kampagne gegen 
die Springer-Presse Ende der 1960er Jahre auch von anderen Medien, unter anderem vom dem ZEIT-Herausgeber Bucerius und dem SPIEGEL-Herausgeber Augstein, finanziell unterstützt worden war. Schneider hatte - neben fünf leitenden Organisatoren des Springer-Tribunals - damals Geld für dessen Durchführung erhalten. Das Institut für Gegenöffentlichkeit (Göfi) war das Dach, unter dem die SpringerArbeitskreise der Studenten ihre Arbeit aufnahmen und Kampagnen gegen die Springer-Presse organisierten. Schneider hatte in dem Interview Bernhard Blanke erwähnt, der richtiggestellt hatte, das sie damals keine 5.000 DM, sondern jeweils nur 500 DM angeboten bekommen hätten - eine Summe, die er damals nicht angenommen hatte. Aber die Diskussion über die angebliche Fremdsteuerung der Springer-Kampagne schlug in den Leitmedien der Republik um diese Zeit erhebliche Wogen. Bernhard Blanke hat sich im Argument (294/2011) ausführlicher hierzu und zu anderen Sachverhalten geäußert. So war sein letztes publizistisches Thema neben der Wulff-Affäre, zu der er sich sehr kritisch geäußert hatte - seine Aktivität in der APO und dann später im SDS. Es entbehrt nicht einer gewissen Ironie, dass ihn am Ende seines Lebens seine Jugend eingeholt hat.

Mit ihm verliert die Politikwissenschaft einen Menschen, der wie nur wenige andere die Geschichte der Bundesrepublik aktiv begleitet hat - als politischer Aktivist in verschiedensten Rollen und als Politikwissenschaftler. In seiner Zeit als Herausgeber beziehungsweise Beiratsmitglied des Leviathan hat er mit dazu beigetragen, dass diese Zeit kritisch reflektiert werden konnte. 